\section{Prospects for a Polarized Electron Source for Next Generation Linear Colliders Based on a SLC-Type Gun*}

H. Tang, J.E. Clendenin, J.C. Frisch, G.A. Mulhollan, D.C. Schultz, and K. Witte

Stanford Linear AcceleratorCenter Stanford University, Stanford, CA 94309

Abstract. The successful operation of a GaAs-based polarized electron source utilizing a DC high voltage gun for the SLC program at SLAC has raised the prospects for a similar source for next generation linear colliders (NGLC). A major challenge in meeting the NGLC requirements is to produce $>10^{12}$ electrons per macrobunch from the cathode. The physics issues that are involved in limiting charge extraction from a GaAs-type cathode and the prospects of realizing a NGLC polarized electron source based on an SLC-type gun will be discussed.

\section{INTRODUCTION}

The SLC has been operating with a polarized electron source since the spring of 1992 [1]. The source produces two 2-ns electron bunches of about $62 \mathrm{~ns}$ apart and with an intensity of up to $8 \times 10^{10}$ electrons/bunch at a repetition rate of 120 $\mathrm{Hz}$. The first bunch of polarized electrons, after being bunched and accelerated but with its intensity significantly reduced (by about $50 \%$ ) due to beam losses, is used to collide with a similar bunch of positrons at the interaction point (IP) with a center of mass energy of about $91 \mathrm{GeV}$ to produce $\mathrm{Z}^{\circ}$ bosons. The second electron bunch, which does not need to be polarized, is used to generate the positron bunch. The polarized electron source uses a DC high voltage gun positron bu $120 \mathrm{kV}$ across its Piece diode structure [2]. The gun uses a thin operated at $120 \mathrm{kV}$ across its Piee diode structure [2]. The gun uses a thin strained $\mathrm{GaAs}(100)$ photocathode, whose surface is treated with cesium and NF to have negative electron affinity (NEA). Coupled with a Nd:YAG pumped Ti:Sapphire laser system that is tuned to the appropriate wavelengths and that has the desired pulse structure, the photocathode gun produces the desired beam for the SLC injector. The performance of the polarized source in all aspects, including reliability, beam polarization and intensity, has been excellent [1]

The successful operation of the SLC polarized source has raised the prospects The successful operation of the SLC polarized source has raised the prospects for a similar polarized source for the next generation of linear colliders (NGLC) [3]. In order to overcome the inherent low repetition rate in a linear collider and also to maximize its energy efficiency, an NGLC requires the use of high-charge macrobunches consisting of about 100 microbunches for the purpose of increased luminosity [3]. The total charge in a single macrobunch is expected to be close to

* Work supported by Department of Energy contract DE-AC03-76SF00515
$1 \times 10^{12}$ electrons at the IP, or at least about $1.5 \times 10^{12}$ electrons at the source which is more than an order of magnitude beyond the present SLC requirement. Although this large amount of charge is distributed over the width of the macrobunch, about $130 \mathrm{~ns}$, and hence the instantaneous current at the source may actually be lower than that for the SLC, the large quantity of the charge in each macrobunch presents the major challenge for developing a NGLC polarized macrobunch presents the major challenge for developing a NGLC polarized
source due to inherent charge-limiting physics that operates in an NEA GaAs source due to inherent charge-limiting physics that operates in an NEA GaAs photocathode. In this paper, we will discuss the prospects of realizing such a
source based on an existing SLC gun from charge considerations only. Other important issues, e.g., laser issues, are beyond the scope of this paper.

\section{CATHODE PHYSICS AND DISCUSSION}

The difficulty of extracting a large number of polarized electrons from a $\mathrm{GaAs}$ photocathode within a short period is due to the charge limit (CL) effect, which was discovered during the course of polarized source research and development at SLAC [4]. The CL effect dictates that the maximum extractable charge from a GaAs cathode is determined by its intrinsic and surface properties (specifically, GaAs cathode is determined by its inticic and is generally lower than the space oping density and surface NEA condition) and is genion charge limit of the gun. In practice, the NEA condition is characterized by the cathode's quantum efficiency $(\mathrm{QE})$, defined as the number of photoemitted lectrons normalized by the number of incident photons.

The data shown in Figures 1 and 2, obtained from two $300 \mathrm{~nm}$ strained $\mathrm{GaAs}(100)$ cathodes of different doping densities with 2-ns excitation laser pulses, illustrate the following important characteristics of the CL effect: (i) once the photoemission is charge limited further increase in the laser pulse energy leads to a monto emitted charge; (ii) for charge limited emission, the charge pulse peaks at an earlier time and shrinks in width due to suppressed emission in the later part of the pulse; (iii) there is a significant interbunch effect, i.e., the CL for a later coming bunch is further decreased by the presence of another at an earlier time; and (iv) the time constant characterizing the decay of the inter-bunch effect depends critically on the doping density.

A simple picture to understand the $\mathrm{CL}$ effect is that electrons excited earlier suppress the emission of subsequently excited electrons within a time scale defined by the decay of the inter-bunch effect. The suppression is most likely caused by an increase in the surface work function due to accumulation of excited electrons at electrons reaching the surface may ultimately escape whereas the majority of them will be trapped at the surface. This mechanism is known as the photovoltaic effect, based on which certain aspects of the CL effect have already been numerically simulated by Herrera-Gomez and Spicer [5]. The data shown in Figure 1 is for a relatively low QE condition so as to emphasize the CL effect. Under the best $\mathrm{QE}$ condition, this cathode is capable of producing $10 \times 10^{10}$ electrons in a 2-ns pulse, approaching what the space charge limit permits. As the cathode's $\mathrm{QE}$ decays with time, the $\mathrm{CL}$ also decreases almost proportionally, and the inter-bunch effect becomes more pronounced [4]. While these results are derived from 2-ns pulse studies, they can be used to help us analyze the feasibility of generating the long NGLC macrobunches. 

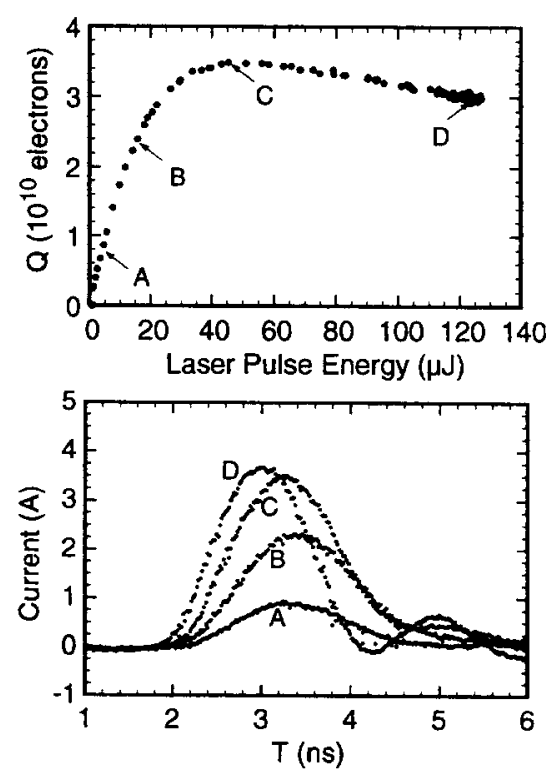

Figure 1: Photoemitted charge versus laser pulse energy (upper panel) and representative charge pulse shapes at various laser pulse energies (lower panel) for a $300 \mathrm{~nm} 5 \times 10^{18}$ $\mathrm{cm}^{-3} \mathrm{Zn}$-doped strained GaAs cathode. The laser wavelength is $865 \mathrm{~nm}$ and pulse width is about $1.8 \mathrm{~ns}$.

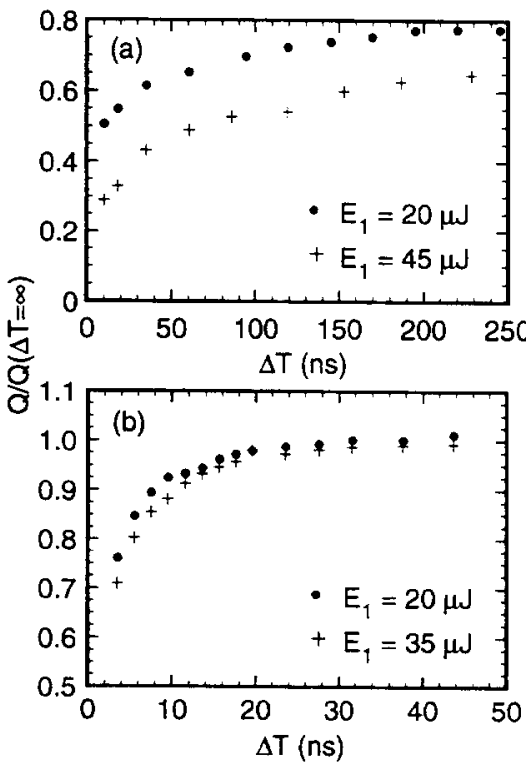

Figure 2: Normalized maximum charge in bunch 2 versus time separation from bunch for (a) a $5 \times 10^{18} \mathrm{~cm}^{-3}$ and (b) a $2 \times 10^{19} \mathrm{~cm}^{-3}$ Zn-doped $300 \mathrm{~nm}$ strained GaAs cathode with bunch 1 just into $C L(\bullet)$ and deep into $C L(t)$ The laser wavelengths for the two bunches are $775 \mathrm{~nm}$ and $865 \mathrm{~nm}$

We will consider the case of using a long, continuous laser pulse to generate an NGLC macrobunch of polarized electrons with a GaAs photocathode based SLC gun [6]. In this scheme, the microbunch structure is created with a bunching system which would admit only about $50 \%$ of the charge in the unchopped macrobunch from the gun. Thus, the total charge in the continuous macrobunch from the gun must be around $3 \times 10^{12}$ electrons, or nearly $4 \mathrm{~A}$ in current over the duration of the pulse. For comparison, the highest operating current out of an SLC polarized gun for the SLC high energy physics program was about $6.5 \mathrm{~A}$, while the space charge limited current is just about twice as much.

A major difference between generating 2-ns and $>100$-ns charge pulses from a $\mathrm{GaAs}$ cathode is that in the former case the cathode may be operated below its CL, as for the 1994 SLC run, while in the latter case the cathode will probably have to be always operated in its CL regime. This is because in the long pulse mode the number of excited electrons accumulated at the cathode's surface will probably inevitably exceed the necessary level for subsequent photoemission to become charge limited. Therefore, the CL effect plays an even more importan role in an NGLC polarized source. In particular, the inter-bunch effect of the CL phenomenon is the most important limiting factor for generating the high-current long charge pulses. This is because it is essentially all of the previously excited electrons over a time duration characteristic of the decay of the inter-bunch effect that contribute to suppressing the emission of subsequently excited electrons. It becomes immediately clear that a short decay time for the inter-bunch effect is very desirable. As it takes a certain time for the CL effect to fully build up and reach a steady state, there will be a transient period at the beginning of the charge pulse during which the intensity will vary with time and consequently may be unusable for the accelerator. The time scale of such a transient period should be comparable to the inter-bunch effect decay time. In what follows we shall discuss semi-quantitatively the possibility of sustaining a $4 \mathrm{~A}$ photoemission out of an SLC gun following the initial transient period.

Let us assume that a cathode has been prepared to have an excellent NEA condition such that an SLC gun equipped with such a cathode is capable of generating a maximum of $1.4 \times 10^{11}$ electrons in a 2 -ns pulse, or about $11 \mathrm{~A}$ of current. This is a realistic assumption since even better performances have been achieved at SLAC. We shall show that the response of the cathode to long intense laser pulses may be inferred from its 2-ns single- and two-bunch CL behaviors.

Take the two cases in Figure 2 for example. For the $5 \times 10^{18} \mathrm{~cm}^{-3}$ doped sample (Figure 2(a)), the inter-bunch effect decay time is on the order of $100 \mathrm{~ns}$. In order to produce the desired current of $4 \mathrm{~A}$, the laser intensity during the long pulse needs to be, say, about $50 \%$ of that of bunch 1 in the upper branch of the data in Figure 2(a), or $50 \mathrm{~kW}(=10 \mu \mathrm{J} / 2 \mathrm{~ns})$ at $77.5 \mathrm{~nm}$ [7]. At this laser intensity, the charge emitted in a 2 -ns pulse should be about $70 \%$ of the maximum, i.e., about $1 \times 10^{11}$ electrons or $8 \mathrm{~A}$. Within one decay time of $100 \mathrm{~ns}$, the total number of excited electrons would be a factor of 25 more than that during the excitation of bunch 1. According to Figure 2(a), as the total number of excited electrons excited during bunch 1 is increased by about a factor of 2, the charge in bunch 2 is suppressed from about $50 \%$ to about $25 \%$ of its single-bunch value at a bunch separation of $10 \mathrm{~ns}$. For a 25 -fold increase in bunch 1 excitation, the charge in bunch 2 is expected to be suppressed to $<10 \%$ of its single-bunch value, i.e., the current in bunch 2 would be below $1 \mathrm{~A}$. Clearly, as this current is well below the desired $4 \mathrm{~A}$ level, an SLC gun using such a GaAs cathode would fail to produce the desired high intensity macrobunch for an NGLC

For the $2 \times 10^{19} \mathrm{~cm}^{-3}$ doped sample (Figure 2(b)), the inter-bunch effect decay time is on the order of $10 \mathrm{~ns}$, one order of magnitude shorter than for the $5 \times 10^{18}$ $\mathrm{cm}^{-3}$ doped sample. All of the above quantitative analysis remains valid except that the 25 -fold increase is now reduced to a mere 2.5 -fold increase. According to Figure 2(b), the charge intensity in bunch 2 at a time separation of $4 \mathrm{~ns}$ from bunch 1 is reduced to about $70 \%$ of its single-bunch value, or about $6 \mathrm{~A}$. Instead of being $4 \mathrm{~ns}$ apart, however, photoemission must proceed continuously in the long macrobunch. This would certainly reduce the steady emission current to below the estimated $6 \mathrm{~A}$, but probably would still exceed the desired $4 \mathrm{~A}$ level. Thus, it appears that an SLC gun using such a cathode might be adequate for use as an NGLC polarized gun.

Of course, the above discussion is solely based on the available data which are obtained from 2-ns pulse studies. It may be regarded as a generalization of the $\mathrm{CL}$ effect from short to long time scales. While we believe that our discussion is quantitatively reasonable, it is possible that new unknown phenomena may arise in going from 2 -ns pulses to $>100$-ns pulses which may complicate our analysis. 
Therefore, the ultimate check of its validity is only possible through experiments. A Q-switched flash-lamp pumped Ti:Sapphire laser system is now under development at SLAC to produce the desired long, high intensity laser pulses. We expect to conduct experiments to study the CL effects on 100-ns time scales toward the end of 1994

\section{OUTLOOK}

The CL effect plays a very important role for the development of an NGLC polarized source. Our analysis suggests that high doping strained $\mathrm{GaAs}$ cathodes hold the best promise for a possible high-polarization electron source for an NGLC. Optimizing a cathode's NEA condition, including the use of larger band gap materials, should always be helpful as it reduces the inter-bunch effect as well as raises the CL. Use of high doping, however, noticeably compromises the polarization obtainable from a strained GaAs cathode due to increased band tailing and other effects. A possible approach to take advantage of the high current capability of high doping cathodes while retaining the high polarization characteristic of low doping cathodes is to use a modulated doping scheme, i.e. high doping in a thin $(50-100 \AA)$ surface layer and low doping in the rest of the active layer. Such a doping scheme has been tested in a superlattice cathode which indeed yielded encouraging results [8]. From the standpoint of charge which indeed yielded encouraging results [8]. From the standpoint of charge considerations, it is fair to stat
for NGLC polarized sources.

\section{REFERENCES}

1. D.C. Schultz, et al., "Polarized source performance in 1992 for SLC-SLD", Proc. of the 10th Intern. Symp. on High Energy Spin Phys., Nagoya, Japan, 1992, p. 833; D.C. Schultz, et al. "The polarized electron source of the Stanford Linear Accelerator Center", SLAC-PUB-6606, 1994, presented at the 17th Interm. Linear Accel. Conf., Tsukuba, Japan, 1994.

2. R.K. Alley, et al., SLAC-PUB-6489, to be published.

3. Specifically, NLC, JC, and SBLC, see e.g. R.H. Siemann, "Linear colliders: the last ten years and the next ten years", Proc. of the Sessler Symp. on Beam Physics, Berkeley, 1993.

4. For details, see M. Woods, et al., J. Appl. Phys. 73, 8531 (1993); H. Tang, et al. "Experimental studies of the charge limit phenomenon in GaAs photocathodes", Proc. of the Workshop on Photocathodes for Polarized Electron Sources for Accelerators, Stanford. CA. 1993, SLAC-432 (Rev), 1994, p. 344.

5. A. Herrera-Gomez and W.E. Spicer, "Physics of high intensity nanosecond electron source", Proc. of 1993 SPIE Intern. Symp. on Optics, Imaging and Instrum., San Diego, 1993, p. 51.

6. The most feasible approach to generate a stable NGLC macrobunch is probably to use a long laser pulse to generate a long charge pulse, and then to create the desired microbunch structure using a bunching system.

7. The wavelength of the laser pulse used for bunch 1 is not important here. If the wavelength used for bunch 1 is to be the same as that for bunch 2 - a case perhaps more appropriate for the discussion on long pulses - the laser pulse energy for bunch 1 simply needs to be larger by about a factor of 3 to drive the photoemission into CL. Also, the exact laser intensity in the long pulse is hard to predict. 50\% appears to be a reasonable assumption.

8. Y. Kurihara, et al., "A high polarization and high quantum efficiency photocathodes using a GaAs-AlGaAs superlattice", SLAC-PUB-6530, submitted to Japn. J. Appl. Phys. 
